\title{
A Ramsey Property of Random Regular and $k$-out Graphs
}

\author{
Michael Anastos* \\ Department of Mathematical Sciences \\ Carnegie Mellon University \\ Pittsburgh, PA; USA
}

\author{
Deepak Bal ${ }^{\dagger}$ \\ Department of Mathematical Sciences \\ Montclair State University \\ Montclair, NJ; USA
}

\begin{abstract}
In this note we consider a Ramsey property of random $d$-regular graphs, $\mathcal{G}(n, d)$. Let $r \geq 2$ be fixed. Then w.h.p. the edges of $\mathcal{G}(n, 2 r)$ can be colored such that every monochromatic component has size $o(n)$. On the other hand, there exists a constant $\gamma>0$ such that w.h.p., every $r$-coloring of the edges of $\mathcal{G}(n, 2 r+1)$ must contain a monochromatic cycle of length at least $\gamma n$. We prove an analogous result for random $k$-out graphs.
\end{abstract}

\section{Introduction}

We are concerned with the following Ramsey-type question: if the edges of a graph are $r$-colored (not necessarily properly), what is the largest monochromatic component (or path, or cycle) which must appear?

This question was considered for Erdős-Rényi random graphs $G(n, p)$ independently by Bohman, Frieze, Krivelevich, Loh and Sudakov [3] and by Spöhel, Steger and Thomas [14]. They proved that for every $r \geq 2$ there is a constant $\psi_{r}$ such that if $c<\psi_{r}-\varepsilon$, then w.h.p. $G(n, c / n)$ admits an $r$-edge coloring where all monochromatic components are of order $o(n)$. If $c>\psi_{r}+\varepsilon$, they prove that w.h.p. every edge coloring contains a monochromatic component of order $\Omega(n)$. The constant $\psi_{r}$ actually arises from the so-called $r$-orientability threshold which was discovered independently by Cain, Sanders and Wormald [5] and by Fernholz and Ramachandran [6]. A graph is called $r$-orientable if its edges can be oriented such that the maximum in-degree of any vertex is at most $r$. Recently, Krivelevich [11] improved upon the results in [3] and [14] by showing that w.h.p. every edge $r$-coloring of $G\left(n, \frac{\psi_{r}+\varepsilon}{n}\right)$ contains not only a linear sized monochromatic component, but actually a linear length monochromatic cycle. The result follows from a nice theorem which proves the existence of long cycles in locally sparse graphs (stated as Theorem 4.1 below).

For regular graphs, Thomassen [15] proved that every 3-regular graph has a 2-coloring of its edges such that every monochromatic component is a path of length at most 5. Alon et. al. [1] proved that every $(2 r-1)$-regular graph can be edge $r$-colored such that each monochromatic component contains at most $120 r-123$ edges. On the other hand, they prove that there exist $2 r$-regular graphs on $n$ vertices such that every edge $r$-coloring contains a monochromatic cycle of length at least $\Omega(\log n)$.

Our first theorem provides an analog of the results of [3], [14] and [11] in the setting of random $d$-regular graphs, $\mathcal{G}(n, d)$.

*manastos@andrew.cmu.edu

${ }^{\dagger}$ deepak. bal@montclair.edu 
Theorem 1.1. For each fixed $r \geq 2$, there exists a constant $\gamma>0$ such that w.h.p.

(i) there exists an r-coloring of the edges of $\mathcal{G}(n, 2 r)$ such that the largest monochromatic component has order o( $n)$;

(ii) every $r$-coloring of the edges of $\mathcal{G}(n, 2 r+1)$ contains a monochromatic cycle of length at least $\gamma n$.

We note that as in the case of binomial random graphs, this "threshold" also corresponds to the orientability threshold for regular graphs. Indeed, a result of Hakimi [8] says that a graph is $r$-orientable if and only if every subgraph has average degree at most $2 r$. Thus $2 r$-regular graphs are $r$-orientable, but $(2 r+1)$-regular graphs are not. Our next theorem provides an analogous result for the model $\mathcal{G}_{n, k \text {-out }}$ where each vertex chooses $k$ random neighbors (see below for the formal definition). Again, this corresponds with the $r$-orientability "threshold."

Theorem 1.2. For each fixed $r \geq 2$, there exists a constant $\gamma>0$ such that w.h.p.

(i) there exists an $r$-coloring of the edges of $\mathcal{G}_{n, r \text {-out }}$ such that the largest monochromatic component has order $o(n)$;

(ii) every $r$-coloring of the edges of $\mathcal{G}_{n,(r+1) \text {-out }}$ contains a monochromatic cycle of length at least $\gamma n$.

In Sections 2 and 3 we prove statement (i) of Theorems 1.1 and 1.2 respectively. In Section 4 , we prove statement (ii) of Theorems 1.1 and 1.2. We conclude in Section 5 with a discussion of a few open problems.

\section{Definitions and Notation}

See [10] or [16] for details on random regular graphs. We use $\mathcal{G}(n, d)$ to refer to a graph drawn uniformly at random from all $r$-regular graphs on vertex set $[n]=\{1, \ldots, n\}$. Further, we refer to two related models: $\mathcal{G}^{*}(n, d)$ and $\mathcal{G}^{\prime}(n, d)$. In the configuration or pairing model, $\mathcal{G}^{*}(n, d)$, a set of nd many configuration points (assuming $n d$ is even) is partitioned into $n$ cells of size $d$, each cell corresponding to a vertex of $[n]$. A perfect matching is placed on the set of configuration points and then each cell is contracted to a vertex resulting in $d$-regular multi-graph in which loops and multi-edges may appear. $\mathcal{G}^{\prime}(n, d)$ is $\mathcal{G}^{*}(n, d)$ conditioned to have no loops. Any property which holds w.h.p. in $\mathcal{G}^{*}(n, d)$ or $\mathcal{G}^{\prime}(n, d)$ also holds w.h.p. in $\mathcal{G}(n, d)$ (see Theorem 9.9 in [10]).

See [7] or [4] for details on random $k$-out graphs. For $1 \leq k \leq n-1$, let $\mathcal{D}_{n, k \text {-out }}$ represent a random digraph on vertex set $[n]$ where each vertex independently chooses a set of $k$ out-neighbors uniformly at random from all $\left(\begin{array}{c}n-1 \\ k\end{array}\right)$ choices. $\mathcal{G}_{n, k \text {-out }}$ is a random (multi)graph obtained from $\mathcal{D}_{n, k \text {-out }}$ by ignoring the orientation of the arcs.

Let $G$ be a (multi)(di)graph. We write $G=G_{1}+\cdots+G_{\ell}$ if i) all of $G, G_{1}, \ldots, G_{\ell}$ are defined on the same vertex set, ii) each of $G_{1}, \ldots, G_{\ell}$ is chosen independently and uniformly at random from a given set of graphs, iii) $E=E(G)=E\left(G_{1}\right) \cup E\left(G_{2}\right) \cup \cdots \cup E\left(G_{\ell}\right)$.

We omit floors and ceilings in certain places for ease of presentation.

\section{Proof of Theorem 1.1 (i)}

It is well known (see Theorem 9.43 of [10]) that if we consider $G=H_{1}+H_{2}+\cdots+H_{r}$ where the $H_{i}$ are chosen from the set of all Hamilton cycles on vertex set $[n]$, then $G$ and $\mathcal{G}^{\prime}(n, 2 r)$ are mutually contiguous and so any property which holds w.h.p. in $G$ also holds w.h.p. in $\mathcal{G}(n, 2 r)$. Thus the following theorem implies Theorem 1.1 (i). 
Theorem 2.1. Let $r \geq 2$ be fixed and $G=H_{1}+H_{2}+\cdots+H_{r}$ be a (multi)graph on $[n]$ where $H_{1}, \ldots, H_{r}$ are chosen from the set of all Hamilton cycles on $[n]$. Then, w.h.p. the edges of $G$ can be $[r]$-colored such that for every $i \in[r]$ the largest component of the graph spanned by the edges of color $i$ has order at most $O\left(n^{0.7}\right)$.

Proof. We reveal $H_{1}$ and we relabel our vertices such that $E\left(H_{1}\right)=\left\{\left\{v_{i}, v_{i+1}\right\}: i \in[n]\right\}$ (we identify $v_{1}$ with $\left.v_{n+1}\right)$. For $i \in\left[n^{0.3}\right]$ set

$$
V_{i}=\left\{v_{j}:\left\lfloor(i-1) n^{0.7}\right\rfloor+1 \leq j \leq\left\lfloor i n^{0.7}\right\rfloor\right\} .
$$

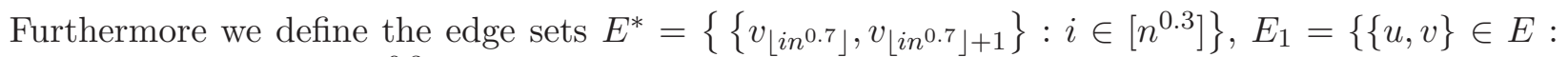
$u, v \in V_{i}$ for some $\left.i \in\left[n^{0.3}\right]\right\}$, and for $i \in[2, r]$ we set $E_{i}=E\left(H_{i}\right) \backslash E_{1}$.

We now implement the following coloring: for $i \in[r]$ we color the edges in $E_{i}$ by color $i$. Additionally we color the edges in $E^{*}$ by color 2 .

Claim 2.2. With probability $1-o(1)$ there does not exist $2 \leq i \leq r$ such that $E_{i}$ spans a path of length larger than $n^{0.4}$.

Proof. Fix $2 \leq i \leq r$ and $v \in V$. Furthermore let $\sigma=\sigma_{i}$ be one of the two permutations associated with $H_{i}$. We consider the exploration of the path $v, \sigma(v), \sigma^{2}(v), \ldots$ induced by $H_{i}$ executed as follows. For $t \in \mathbb{N}$ given the path $v, \sigma(v), \cdots, \sigma^{t-1}(v)$ we query $\sigma^{t}(v)$. We define the stopping time of the exploration to be $\tau=\min \left\{t:\left\{\sigma^{t-1}(v), \sigma^{t}(v)\right\} \in E_{1}\right\}$.

Now let $1 \leq t \leq n^{0.4}$ and let $z_{t} \in\left[n^{0.3}\right]$ be such that $\sigma^{t-1}(v) \in V_{z_{t}}$. Furthermore assume that we have not stopped the exploration of the path at time $t-1$ i.e. $\tau>t-1$. Then $\sigma^{t}(v)$ is uniformly distributed in $V \backslash\left\{v, \sigma(v), \ldots, \sigma^{t-1}(v)\right\}$. Thus $\sigma^{t}(v) \in V_{z t}$ with probability at least $\frac{\left|V_{z_{t}}\right|-t}{n-t} \geq \frac{0.9 n^{0.7}}{n}$. Therefore

$$
\mathbb{P}\left(\tau>n^{0.4}\right)=\prod_{j=1}^{n^{0.4}} \mathbb{P}(\tau>j \mid \tau>j-1) \leq \prod_{j=1}^{n^{0.4}}\left(1-\frac{0.9}{n^{0.3}}\right) \leq e^{-n^{0.4} \cdot \frac{0.9}{n^{0.3}}}=e^{-0.9 n^{0.1}}
$$

Thus the probability that the subpath of $v, \sigma(v), \sigma^{2}(v), \ldots$ that is incident to vertex $v$ and is induced by the edges in $E_{i}$ is larger than $n^{0.4}$ is less than $e^{-0.9 n^{0.1}}=o\left(1 / n^{2}\right)$. Taking a union bound over all $2 \leq i \leq r$ and $v \in V$, we find that every segment of length $n^{0.4}$ of each $H_{i}$ contains an edge of $E_{1}$. Thus each $E_{i}, i \geq 2$ consists of disjoint paths of length less than $n^{0.4}$

Observe that the largest component of the graph spanned by $E_{1}$ is spanned by some $V_{j}, j \in\left[n^{0.3}\right]$ and therefore it has size $\left\lceil n^{0.7}\right\rceil$. For $2 \leq i \leq r, E_{i}$ is the union of vertex disjoint paths each of which w.h.p. has length at most $n^{0.4}$ (see Claim 2.2). Thus the largest component spanned by color $i$, for $3 \leq i \leq r$ is of size at most $n^{0.4}$. Finally we colored by color 2 the edges in $E_{2} \cup E^{*}$. Hence, since $\left|E^{*}\right|=n^{0.3}$ and any component spanned by $E_{2}$ has size at most $n^{0.4}$ we have that the largest component spanned by the edges of color 2 has w.h.p. size at most $\left(n^{0.3}+1\right) \cdot n^{0.4}$.

\section{Proof of Theorem $1.2(\mathrm{i})$}

Let $D$ be the directed graph $D=D_{1}+D_{2}+\ldots+D_{k}$ where $D_{i}$ is chosen from all directed graphs on $V$ where every $v \in V$ has out-degree 1 (we forbid loops) and $|V|=n$. Then with probability bounded away from zero, every vertex in $D$ has $k$ distinct neighbors and we obtain $\mathcal{D}_{n, k \text {-out }}$. Let 
$G$ be the (multi)graph obtained by ignoring the orientation of the $\operatorname{arcs}$ in $D$. Then we have that $\mathcal{G}_{n, k \text {-out }}$ is contiguous with respect to $G$, i.e. every statement which holds w.h.p. in $G$ also holds w.h.p. in $\mathcal{G}_{n, k \text {-out }}$ Thus the following theorem implies Theorem 1.2 (i).

Theorem 3.1. Let $r \geq 2$ be fixed and let $D=D_{1}+D_{2}+\ldots+D_{r}$ be a (multi)digraph where $D_{i}$ is chosen from all directed graphs on $[n]$ where every $v \in[n]$ has out-degree 1 . Let $G$ be the (multi)graph obtained by ignoring the orientations of the arcs in D. Then, w.h.p. the edges of $G$ can be $[r]$-colored such that for every $i \in[r]$ the largest component of the graph spanned by the edges of color $i$ has order at most $O\left(n^{0.9}\right)$.

Proof. We start by constructing a partition of $V$ into $(1+o(1)) n^{0.1}$ sets each of size $(1+o(1)) n^{0.9}$ and finding a set $E^{*} \subset E\left(D_{1}\right)$ of size at most $n^{0.2}$ such that if an arc in $E\left(D_{1}\right)$ has its endpoints in different sets of the partition then it belongs to $E^{*}$.

We construct the partition and $E^{*}$ as follows. Remove one arc from each cycle of $D_{1}$, and add it to $E^{*}$. Observe that in expectation $D_{1}$ has $O(\log n)$ cycles (see e.g. Section 14.5 of [4) hence w.h.p. we have added at most $n^{0.1}$ arcs to $E^{*}$. At the same time the removal of those arcs turns $D_{1}$ into the union of vertex disjoint in-arborescences. Henceforward we implement the following algorithm

While $D_{1}$ contains an in-arborescence of order larger than $n^{0.85}$ :

- Pick a vertex $v$ such that in $D_{1}, v$ is reachable by at least $n^{0.85}$ vertices but none of $v$ 's in-neighbors have this property.

- Remove every in-arc of $v$ from $D_{1}$ and add it to $E^{*}$.

The maximum in-degree of $D_{1}$ is w.h.p. less than $\log n$. Therefore at every iteration we add to $E^{*}$ at most $\log n$ arcs. Moreover after each iteration at least $n^{0.85}$ additional vertices are spanned by in-arborescences of size at most $n^{0.85}$. Therefore there are at most $n^{0.15}$ iterations and w.h.p. $\left|E^{*}\right| \leq n^{0.15} \log n+n^{0.1}<n^{0.2}$.

The removal of $E^{*}$ breaks $D_{1}$ into in-arborescences $A_{1}, \ldots, A_{\ell}$ each of size at most $n^{0.85}$. For $1 \leq j<n^{0.1}$ define $h_{j}=\min \left\{i \in[\ell]:\left|\cup_{d \leq i} V\left(A_{d}\right)\right| \geq j n^{0.9}\right\}$. Also set $h_{0}=0$ and $h_{n^{0.1}}=\ell$. Partition $V$ into $V_{1}, \ldots, V_{n^{0.1}}$, where $V_{j}=\cup_{h_{j-1}<b \leq h_{j}} V\left(A_{b}\right)$. Finally define $E_{1}=\{(u, v) \in E(D)$ : $u, v \in V_{j}$ for some $\left.j \in\left[n^{0.1}\right]\right\}$, and for $i \in[2, r]$ we set $E_{i}=E\left(D_{i}\right) \backslash E_{1}$.

We now implement the following coloring of the edges of $G$ : for $i \in[r]$ we color the edges of $G_{k}$ obtained from $E_{i}$ by color $i$. Additionally we color the edges obtained from $E^{*}$ by color 2 .

Claim 3.2. With probability $1-o(1)$ there does not exist $2 \leq i \leq r$ such that $E_{i}$ spans a path of length larger than $n^{0.15}$.

Proof. Fix $2 \leq i \leq r$ and $v \in V$. Furthermore let $f=f_{i}$ be a function $f: V \rightarrow V$ such that $E\left(D_{i}\right)=\{(v, f(v)): v \in V\}$. We consider the exploration of the walk $v, f(v), f^{2}(v), \ldots$ induced by $E_{i}$ executed as follows. For $t \in \mathbb{N}$ given the walk $v, f(v), \cdots, f^{t-1}(v)$ we query $f^{t}(v)$. We define the stopping time of the exploration to be

$$
\tau=\min \left\{t:\left(f^{t-1}(v), f^{t}(v)\right) \in E_{1} \quad \text { or } \quad f^{t}(v)=f^{i}(v) \text { for some } i<t\right\} .
$$

Now let $1 \leq t \leq n^{0.15}$ and let $z_{t} \in\left[n^{0.1}\right]$ be such that $f^{t-1}(v) \in V_{z t}$. Furthermore assume that we have not stopped the exploration of the walk at time $t-1$ i.e. $\tau>t-1$. Then $f^{t}(v)$ has not 
yet been exposed and is uniformly distributed in $V \backslash\left\{f^{t-1}(v)\right\}$. Thus $f^{t}(v) \in V_{z_{t}}$ with probability $\frac{\left|V_{z_{t}}\right|-1}{n-1} \geq \frac{0.9 n^{0.9}}{n}$. Therefore

$$
\mathbb{P}\left(\tau>n^{0.15}\right)=\prod_{j=1}^{n^{0.15}} \mathbb{P}(\tau>j \mid \tau>j-1) \leq \prod_{j=1}^{n^{0.15}}\left(1-\frac{0.9}{n^{0.1}}\right) \leq e^{-n^{0.15} \cdot \frac{0.9}{n^{0.1}}} \leq \frac{1}{n^{2}}
$$

Thus the probability that the sub-walk of $v, f(v), f^{2}(v), \ldots$ incident to $v$ and induced by $E_{i}$ has length larger than $n^{0.15}$ is less than $1 / n^{2}$. Taking a union bound over all $2 \leq i \leq k$ and $v \in V$ the claim follows.

Claim 3.3. With probability $1-o(1)$ there does not exist $2 \leq i \leq r$ such that $E\left(D_{i}\right)$ spans an in-arborescence of height at most $n^{0.15}$ and order greater than $\bar{n}^{0.7}$.

Proof. Let $2 \leq i \leq r, v \in V$. We explore the in-arborescence rooted at $v$ in $D_{i}$ using breadthfirst search. For $j \geq 0$ let $\ell_{j}$ be the number of vertices at level $j$ (where level 0 contains only $v)$. Furthermore let $t_{j}=\sum_{0 \leq i \leq j} \ell_{i}$. Then, given $\ell_{0}, \ldots, \ell_{h}$ we have that $\ell_{h+1}$ is distributed as $\operatorname{Bin}\left(n-t_{h}, \frac{\ell_{h}}{n-t_{h-1}}\right)$ i.e a binomial random variable with $n-t_{h}$ trials and probability of success $\ell_{h} /\left(n-t_{h-1}\right)$. Hence $\ell_{h+1}$ is dominated by $\operatorname{Bin}\left(n_{h}, \ell_{h} / n_{h}\right)$ where $n_{h}=n-t_{h-1}$. Observe that using the Chernoff bound, i.e. $\mathbb{P}[\operatorname{Bin}(n, p) \geq(1+\epsilon) n p] \leq \exp \left\{-\epsilon^{2} n p / 3\right\}$ (see e.g. [10]), for any $h \in[n]$ we have

$$
\mathbb{P}\left[\ell_{h+1}>2 n^{0.51} \mid \ell_{h}<n^{0.51}\right] \leq \mathbb{P}\left[\operatorname{Bin}\left(n_{h}, \frac{n^{0.51}}{n_{h}}\right)>2 n^{0.51}\right] \leq \exp \left\{-n^{0.51} / 3\right\} .
$$

Furthermore

$$
\begin{aligned}
\mathbb{P}\left[\ell_{h+1}>\left(1+n^{-0.25}\right) \ell_{h} \mid \ell_{h}>n^{0.51}\right] & \leq \mathbb{P}\left[\ell_{h+1}>\left(1+\frac{n^{0.05}}{\ell_{h}^{0.5}}\right) \ell_{h} \mid \ell_{h}>n^{0.51}\right] \\
& \leq \mathbb{P}\left[\operatorname{Bin}\left(n_{h}, \ell_{h} / n_{h}\right)>\left(1+\frac{n^{0.05}}{\ell_{h}^{0.5}}\right) \ell_{h}\right] \leq \exp \left\{-n^{0.1} / 3\right\}
\end{aligned}
$$

Therefore with probability at least $1-o(1 / n)$ for every $h \in\left[n^{0.15}\right]$ we have that $\ell_{h} \leq(1+$ $\left.n^{-0.25}\right)^{h} 2 n^{0.51} \leq 4 n^{0.51}$. Thus with probability at least $1-o(1 / n)$ in $D_{i}$ any in-arborescence of height at most $n^{0.15}$ rooted at $v$ spans less than $n^{0.15} \cdot 4 n^{0.51} \leq n^{0.7}$ vertices. By taking a union bound over all $2 \leq i \leq r$ and $v \in V$ the claim follows.

Now observe that the largest component of the graph spanned by the edges obtained from $E_{1}$ is contained in some $V_{j}, j \in\left[n^{0.1}\right]$ and therefore the largest component of color 1 has size at most $(1+o(1)) n^{0.9}$. Now consider $E_{i}$ for $2 \leq i \leq r$. Each component of the digraph induced by $E_{i}$ is either an in-arborescence or unicyclic in which case we can view it as an in-arborescence plus an edge. By Claim 3.2. each such component (viewed as an in-arborescence) has height at most $n^{0.15}$ and thus by Claim 3.3 has order at most $n^{0.7}$. Thus the largest component spanned by color $i$, $3 \leq i \leq r$, is of order at most $n^{0.7}$. Finally the largest component spanned by edges obtained from $E_{2}$ is of size $n^{0.7}$ and we have $\left|E^{*}\right| \leq n^{0.2}$. Therefore the largest component spanned by color 2 is of order $O\left(n^{0.9}\right)$. 


\section{Proof of Theorem 1.1 (ii) and Theorem 1.2 (ii)}

This section follows closely the recent paper of Krivelevich [11]. We begin by stating a Theorem from [11] which we will use. This theorem says that graphs with decent global density, but relatively smaller local density must contain a long cycle.

Theorem 4.1 (Theorem 2 of [11]). Suppose reals $c_{1}>c_{2}>1$ and a positive integer $k$ satisfy $\left(\frac{k}{2}-1\right)\left(\left(\frac{c_{1}}{c_{2}}\right)^{1 / 2}-1\right) \geq 2$. Let $G=(V, E)$ be a graph on at least $k$ vertices satisfying

(i) $|E(G)| \geq c_{1}|V|$

(ii) every subset $S \subseteq V$ of size $|S| \leq k$ satisfies $e(S) \leq c_{2}|S|$.

Then $G$ contains a cycle of length at least $\left(\frac{k}{2}-1\right)\left(\left(\frac{c_{1}}{c_{2}}\right)^{1 / 2}-1\right)$.

The following lemma (whose proof is almost identical to that of Proposition 3 of [11]) verifies that random regular graphs satisfy the "local sparseness" condition (ii) of Theorem 4.1

Lemma 4.2. Let $d \geq 2$ be an integer, let $d>c>1$ and let $\delta=\left(\frac{1}{3} \cdot \frac{c^{c}}{e^{1+c} d^{c}}\right)^{\frac{1}{c-1}}$. Then, w.h.p. every subset $S$ of $\mathcal{G}(n, d)$ of size $|S| \leq \delta n$ satisfies $e(S) \leq c|S|$.

Proof. The probability that there is a subset $S$ in $\mathcal{G}^{*}(n, d)$ with $|S| \leq \delta n$ and $e(S)>c k$ is at most

$$
\sum_{k \leq \delta n}\left(\begin{array}{l}
n \\
k
\end{array}\right)\left(\begin{array}{l}
d k \\
c k
\end{array}\right)\left(\frac{d k}{d n}\right)^{c k} \leq \sum_{k \leq \delta n}\left(\frac{n e}{k}\right)^{k}\left(\frac{d k e}{c k}\right)^{c k}\left(\frac{k}{n}\right)^{c k} \leq \sum_{k \leq \delta n}\left[\frac{e^{1+c} d^{c}}{c^{c}}\left(\frac{k}{n}\right)^{c-1}\right]^{k}
$$

To get the first expression, we choose $k$ cells corresponding to $S$, then we choose $c k$ configuration points within those $d k$ configuration points. $\frac{d k}{d n}$ is a bound on the probability that one of these $c k$ points matches to one of the $d k$ points corresponding to $S$. Let $u_{k}=\left[\frac{e^{1+c} d^{c}}{c^{c}}\left(\frac{k}{n}\right)^{c-1}\right]^{k}$. If $k \leq \ln n$, then $u_{k} \leq\left[O(1)\left(\frac{\ln n}{n}\right)^{c-1}\right]^{k}$, so $\sum_{k \leq \ln n} u_{k}=o(1)$. If $\ln n<k \leq \delta n$, then using the value of $\delta$, we get

$$
u_{k} \leq\left[\frac{e^{1+c} d^{c}}{c^{c}} \delta^{c-1}\right]^{k}=\frac{1}{3^{k}}=o(1 / n)
$$

and so w.h.p. there is no subset violating the property.

Proof of Theorem 1.1 (ii). Let $G \sim \mathcal{G}(n, 2 r+1)$. Then Lemma 4.2 applied to $G$ with $d=2 r+1$ and $c=1+\frac{1}{4 r}$ implies that every subset $S$ of size $|S| \leq \delta n$ has $e(S) \leq\left(1+\frac{1}{4 r}\right)|S|$. Note that this property is inherited by any subgraph of $G$.

Let the edges of $G$ be $r$-colored and let $\widehat{G}$ be the subgraph whose edges are the majority color. Then $|E(\widehat{G})| \geq \frac{1}{r}|E(G)|=\frac{1}{r} \frac{2 r+1}{2} n=\left(1+\frac{1}{2 r}\right) n$. Thus Theorem 4.1 applied to $\widehat{G}$ with $c_{1}=1+\frac{1}{2 r}$, $c_{2}=1+\frac{1}{4 r}$ and $k=\delta n$ implies that $\widehat{G}$ has a cycle (and thus $G$ has a monochromatic cycle) of length at least

$$
\left(\frac{\delta n}{2}-1\right)\left(\left(\frac{1+\frac{1}{2 r}}{1+\frac{1}{4 r}}\right)^{1 / 2}-1\right) \geq \gamma n
$$

for appropriate $\gamma>0$. 
Proof Sketch of Theorem 1.2 (ii). The proof that $\mathcal{G}_{n, d \text {-out }}$ satisfies Lemma 4.2 is essentially the proof for $\mathcal{G}(n, d)$ verbatim. Since $\left|E\left(\mathcal{G}_{n,(r+1) \text {-out }}\right)\right|=(r+1) n$ we may apply Theorem 4.1 to the graph induced by the majority color with $c_{1}=1+\frac{1}{r}, c_{2}=1+\frac{1}{2 r}$ and $k=\delta n$ to complete the proof as above.

\section{Conclusion}

We note that much of the work related to this problem concerns vertex colorings rather than edge colorings. See [1, 2, 9, 12, 13]. Coloring the vertices of a graph such that each color class induces only small components is a natural relaxation of proper coloring. It would be very interesting to consider these "bounded monochromatic component" problems in the context of random regular graphs. As just one example, in 9] it is proved that every 4-regular graph $G$ has a vertex partition $V=V_{1} \cup V_{2}$ such that $G\left[V_{1}\right]$ and $G\left[V_{2}\right]$ contain only components of order at most 6 . It is also noted that in general 6 cannot be replaced by a number less than 4 . One can ask for the best number which can be used if restricting attention to random 4-regular graphs.

In this note, we have shown that almost every $2 r$-regular graph admits an $r$-edge-coloring where every component has $O\left(n^{0.7}\right)$ many vertices. Our argument can be improved to give $O\left(n^{2 / 3+o(1)}\right)$ but an obvious open problem is to improve this upper bound for random $2 r$-regular graphs. From the algorithmic side, we ask the following question.

Problem 5.1. Does there exist a polynomial time algorithm which $r$-colors the edges of a random $2 r$-regular graph such that w.h.p. every monochromatic component is of order o $(n)$ ?

We note that our proof could solve this problem if one could find an algorithm which decomposes a random $2 r$-regular graph into $r$ Hamilton cycles such that the probability that the algorithm outputs a "bad" $r$-tuple of Hamilton cycles i.e. one that does not satisfy Claim 2.2, is $o(1)$.

Another extension concerns online version of the above problem. Let $e_{1}, e_{2}, \ldots, e_{\tau}$ be a random permutation of the edges of $\mathcal{G}(n, 2 r)$. For $1 \leq i \leq \tau$ at step $i$ the edge $e_{i}$ is revealed. The objective is to find an algorithm $\mathcal{A}$ that runs in polynomial time which, on step $i$, assigns a color from $[r]$ to $e_{i}$ without any knowledge of $e_{i+1}, \ldots, e_{\tau}$. $\mathcal{A}$ must maintain w.h.p. that the size of every monochromatic component is $o(n)$ until all edges have been revealed. In [3], Bohman et. al. consider both the online and the offline version for $G(n, p)$. However the ranges of $p$ for which they proved that $G(n, p)$ can be $r$-colored such that w.h.p. every monochromatic component is of order $o(n)$ differ in the two settings.

A final interesting problem is to determine the best bound for arbitrary $2 r$-regular graphs. Alon et. al. [1] proved that every $(2 r-1)$-regular graph admits an $r$-edge-coloring with bounded size components whereas Theorem 1.1(ii) shows that there exist $(2 r+1)$-regular graphs such that every coloring contains a linear order component (actually cycle). The following problem is essentially posed in 12 for $r=2$, but we state it here.

Problem 5.2. Given $r \geq 2$, what is the smallest integer $f_{r}(n)$ such that every $2 r$-regular graph on $n$ vertices admits an r-edge-coloring where all components have order at most $f_{r}(n)$ ? Is $f_{r}(n)$ sublinear?

The construction in [1] provides a lower bound of $f_{r}(n)=\Omega(\log n)$. Perhaps the consideration of random $2 r$-regular graphs could lead to an improvement of this lower bound. 


\section{References}

[1] Noga Alon, Guoli Ding, Bogdan Oporowski, and Dirk Vertigan. Partitioning into graphs with only small components. J. Combin. Theory Ser. B, 87(2):231-243, 2003.

[2] Robert Berke and Tibor Szabó. Relaxed two-coloring of cubic graphs. J. Combin. Theory Ser. B, 97(4):652-668, 2007.

[3] Tom Bohman, Alan Frieze, Michael Krivelevich, Po-Shen Loh, and Benny Sudakov. Ramsey games with giants. Random Structures Algorithms, 38(1-2):1-32, 2011.

[4] Béla Bollobás. Random graphs, volume 73 of Cambridge Studies in Advanced Mathematics. Cambridge University Press, Cambridge, second edition, 2001.

[5] Julie Anne Cain, Peter Sanders, and Nick Wormald. The random graph threshold for $k$ orientability and a fast algorithm for optimal multiple-choice allocation. In Proceedings of the Eighteenth Annual ACM-SIAM Symposium on Discrete Algorithms, pages 469-476. ACM, New York, 2007.

[6] Daniel Fernholz and Vijaya Ramachandran. The $k$-orientability thresholds for $G_{n, p}$. In Proceedings of the Eighteenth Annual ACM-SIAM Symposium on Discrete Algorithms, pages 459-468. ACM, New York, 2007.

[7] A. Frieze and M. Karoński. Introduction to Random Graphs. Cambridge University Press, 2015 .

[8] S. L. Hakimi. On the degrees of the vertices of a directed graph. J. Franklin Inst., 279:290-308, 1965 .

[9] Penny Haxell, Tibor Szabó, and Gábor Tardos. Bounded size components-partitions and transversals. J. Combin. Theory Ser. B, 88(2):281-297, 2003.

[10] Svante Janson, Tomasz Łuczak, and Andrzej Rucinski. Random graphs. Wiley-Interscience Series in Discrete Mathematics and Optimization. Wiley-Interscience, New York, 2000.

[11] Michael Krivelevich. Long cycles in locally expanding graphs, with applications. manuscript. arXiv:1609.06851, 2017.

[12] Nathan Linial, Jiří Matoušek, Or Sheffet, and Gábor Tardos. Graph colouring with no large monochromatic components. Combin. Probab. Comput., 17(4):577-589, 2008.

[13] Jiří Matoušek and Aleš Př́ivětivý. Large monochromatic components in two-colored grids. SIAM J. Discrete Math., 22(1):295-311, 2008.

[14] Reto Spöhel, Angelika Steger, and Henning Thomas. Coloring the edges of a random graph without a monochromatic giant component. Electron. J. Combin., 17(1):Research Paper 133, 7,2010 .

[15] Carsten Thomassen. Two-coloring the edges of a cubic graph such that each monochromatic component is a path of length at most 5. J. Combin. Theory Ser. B, 75(1):100-109, 1999.

[16] N. C. Wormald. Models of random regular graphs. In Surveys in combinatorics, 1999 (Canterbury), volume 267 of London Math. Soc. Lecture Note Ser., pages 239-298. Cambridge Univ. Press, Cambridge, 1999. 